\title{
Short and Ultrashort Pulsed Laser Processing of Zinc: Resolidification Morphology of Ablated Craters
}

\author{
H. Mustafa*, M. Jalaal**, W. Ya*, N. Ur Rahman*, D.T.A. Matthews*,***,**** and G.R.B.E. Romer* \\ * Chair of Laser Processing, Department of Mechanics of Solids, Surfaces \& Systems (MS3), \\ Faculty of Engineering Technology, University of Twente, Enschede, the Netherlands \\ E-mail: h.mustafa@utwente.nl \\ ** Physics of Fluids Group and Max Planck Center Twente, MESA+ Institute and J. M. Burgers \\ Centre for Fluid Dynamics, Faculty of Science and Technology, University of Twente, the \\ Netherlands \\ *** Chair of Skin Tribology, Department of Mechanics of Solids, Surfaces \& Systems (MS3), \\ Faculty of Engineering Technology, University of Twente, Enschede, the Netherlands \\ **** Tata Steel Research and Development, IJmuiden, the Netherlands
}

\begin{abstract}
Laser surface texturing of polycrystalline zinc in bulk form and as galvanised coatings is performed with single, as well as multiple nanosecond and picosecond laser pulses at a wavelength of $1030 \mathrm{~nm}$. By varying the laser fluence over a wide range, as well as varying the number of pulses on the same location, thousands of ablated craters can be fabricated and their morphology studied. From the analysis thereof, it can be concluded that, within the boundaries of our experimental (laser) conditions, ablated craters always show molten features. Depending on the pulse fluence and duration, the final solidifed morphology can feature jets with meso- and micro scale spherical endings, micro rims and protrusions, as well as nano-roughness or a rim around the ablated crater. If the laser fluence is high, one observes the evidence of surface waves and finger formation, resulted from different types of hydrodynamic instablity. In contrast to nanosecond laser processing, it is found that craters formed using picosecond pulses deviate significantly from the Gaussian shape of the laser intensity distribution at high laser intensity, due to different temperature gradients in the electronic and lattice subsystems.
\end{abstract}

DOI: 10.2961/jlmn.2018.03.0006

Keywords: ultrashort laser, ablation, surface morphology, polycrystalline zinc, coated steel, splash, hump, rim

\section{Introduction}

Zinc (Zn) is a widely used metal in galvanizing and alloying, primarily for its excellent cathodic protection and corrosion resistance properties [1]. To improve surface functionality, (ultra) short pulsed laser-surface texturing can be used to add functionalities like superhydrophobicity, oleophilicity, and/or improve functionalities like corrosion performance and visual appearance to the Zn layer on steel [2, 3]. Short and ultrashort pulsed laser processing of $\mathrm{Zn}$ is mainly aimed at nanoparticle generation [4, 5], whereas the resulting surface morphology and properties are of more interest than the ablated material for surface texturing applications.

If the time between consecutive pulses is long enough during multi-pulse processing, the laser irradiated surface undergoes rapid solidification after the laser pulse. However, the solidified features depend on the pulse duration of the laser source because of the ablation processes associated with nanosecond (ns) and femtosecond (fs) laser pulses. In ultrashort ( $\tau<1 \mathrm{ps}$ ) pulsed laser material interaction, the material undergoes absorption of laser light, ballistic electron generation, lattice thermalization, subsequent phase changes through spallation, phase explosion, evaporation and finally residual energy dissipation [6]. On the other hand, the difference in the temperature gradients in electronic and lattice subsystem vanishes in short ( $\tau>1 \mathrm{ns)}$ pulsed laser material interaction [7]. This results in vaporization of the material, generation of plasma plume and subsequent hydrodynamic motion of the melt exerted by the recoil pressure of the plasma plume [8]. Thus, the solidification time of the molten volume in ultrashort pulsed laser irradiation is shorter than short pulsed laser irradiation. This results in different resolidified surface structures resulting either from local hydrodynamic instabilities (e.g. Kelvin Helmholtz instability [9]) or from overall thermocapillary motion of the liquid (e.g. Marangoni flow).

In this paper, we investigate the resolidified surface structures of laser-textured surfaces of polycrystalline $\mathrm{Zn}$ in bulk form and as galvanized coatings irradiated by single and multiple picosecond and nanosecond laser pulses at a wavelength of and around $1030 \mathrm{~nm}$ over a wide range of laser fluence levels. Surface morphology of thousands of ablated craters is studied in depth with confocal laser microscopy, atomic force microscopy and scanning electron microscopy.

\section{Experimental Setup \\ 2.1 Laser setup}

The ablation experiments were performed under atmospheric conditions in a cleanroom environment using two different laser sources as listed in Table 1. Both the laser 


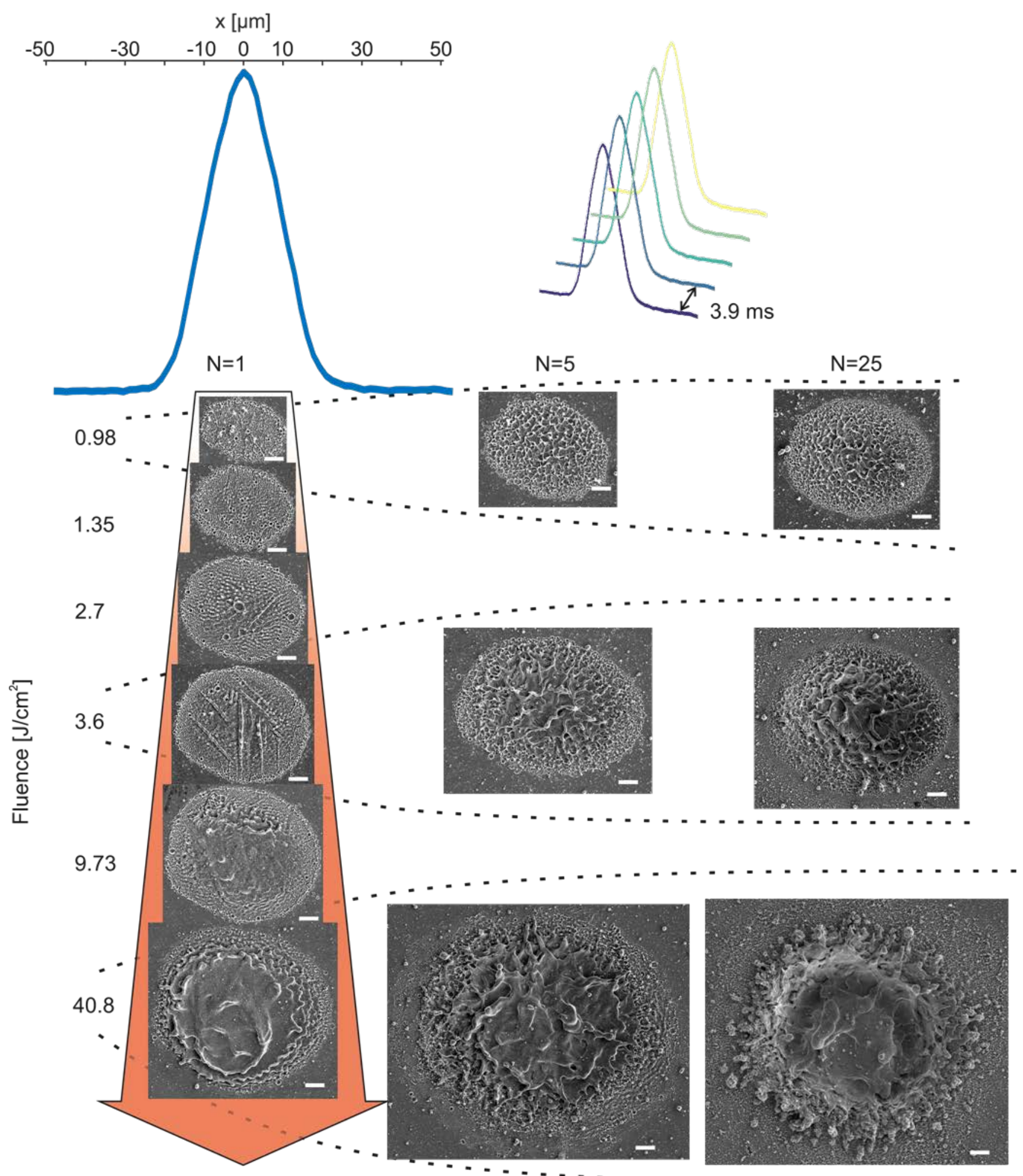

Fig. 1 Laser beam cross-section (top-left) and SEM images (top view) of zinc surface irradiated at different laser peak fluence $F_{0}$ levels at $N=1,5$ and 25 for 7 ps laser pulses (bottom). All images are in same scale. White bar in the SEM images denotes $5 \mu \mathrm{m}$.

sources show a nearly Gaussian power density profile $\left(\mathrm{M}^{2}<\right.$ 1.3). A galvo-scanner (IntelliScan14 of ScanLab GmbH, Germany), equipped with a telecentric flatfield F-thetaRonar lens (Linos GmbH, Germany) of $80 \mathrm{~mm}$ focal length, was used to scan the focus of the laser beam over the surface of the sample. The sample was placed in the focal plane. The focal spot radius of both the sources were measured using a charge-coupled device (CCD) sensor-based, beam diagnostic system (MicroSpot Monitor of Primes GmbH, Germany). The beam was impinging perpendicular to the sample surface. The laser energy supplied to the surface was varied by using a combination of a half-wave plate and a linear polarizer. A pyroelectric detector (PM30 with FieldMax II of Coherent, USA) was used to measure the average laser power 
incident on the sample. The laser-induced surface profiles are referred to as 'craters' in the forthcoming sections. At a repetition rate of $8 \mathrm{kHz}$ and a beam scanning velocity of 1 $\mathrm{m} / \mathrm{s}$, time between consecutive pulses on the same location was at least $3.9 \mathrm{~ms}$. The geometrical pulse-to-pulse distance was at least $125 \mu \mathrm{m}$ and the number of pulses varied from $N$ $=1$ to 50. A minimum of 21 craters were created per laser setting to get statistically sound values.

Table 1 Laser sources used in this work

\begin{tabular}{lll}
\hline Parameter & $\begin{array}{l}\text { Trumpf Tru- } \\
\text { Micro 5050 }\end{array}$ & $\begin{array}{l}\text { SPI redEnergy } \\
\text { G4 }\end{array}$ \\
\hline Pulse duration & $6.7 \mathrm{ps}$ & 3 to $500 \mathrm{~ns}$ \\
Wavelength [nm] & 1030 & 1060 \\
Output power instability [\%] & $<2$ & $<5$ \\
Focal spot radius [ $\mu \mathrm{m}]$ & $14.6 \pm 1.6$ & $15.2 \pm 1.6$ \\
Ellipticity of focal spot & 0.89 & 0.81 \\
\hline
\end{tabular}

\subsection{Material}

Typical zinc (99.7\%wt Zn, 0.3\%wt Al) used for coating on steel products, was melted in a crucible at $460^{\circ} \mathrm{C}$ and cast by gradual cooling in a ceramic crucible. Then the sample was sectioned followed by compression mounting in phenolic resin for metallographic analysis. Preparation procedures included grinding with SiC emery paper (18 $\mu \mathrm{m}$ and $10 \mu \mathrm{m}$ grit size), polishing with 1 and $3 \mu \mathrm{m}$ diamond suspension and final polishing with colloidal silica $(0.04 \mu \mathrm{m})$ suspension. After polishing, an average roughness $\left(R_{a}\right)$ lower than $30 \mathrm{~nm}$ was measured by confocal laser microscopy. The polished polycrystalline zinc sample had a minimum grain size of about $200 \mu \mathrm{m}$. The coated samples (galvanized steel) with a surface roughness $\left(R_{a}\right)$ of $0.3 \mu \mathrm{m}$ was commercially produced according to European standard EN10346:2015 and has a nominal Zn layer thickness of 10 $\mu \mathrm{m}$. These samples were cleaned using Ammonia $(<5 \%)$ solution prior to and after the ablation experiments.

\subsection{Analysis tools}

Craters were measured by means of Confocal Laser Scanning Microscopy (CLSM), (VK-9700 of Keyence Corporation, Japan). The lateral and vertical resolution of CLSM measurements were $276 \mathrm{~nm}$ and $1 \mathrm{~nm}$, respectively. The crater morphology was also analyzed by means of a field emission Scanning Electron Microscope (SEM), (JSM$7200 \mathrm{~F}$ of Jeol, Japan) as well as Atomic Force Microscopy(AFM), (NX10 of Park Systems Corporation, South Korea). The energy-dispersive X-ray spectroscopy (EDS), (X - Max ${ }^{\mathrm{N}}$ of Oxford Instruments, USA) analysis was conducted with the same SEM with an accelerating voltage of 3 $\mathrm{kV}$ for an $\mathrm{X}$-ray generation volume close to the surface.

\section{Results \& Discussions}

First, this section describes the evolution of the ablated crater morphology with the incident number of pulses ranging from 1 to 50 and laser fluences ranging from $0.98 \mathrm{~J} / \mathrm{cm}^{2}$ to $40.8 \mathrm{~J} / \mathrm{cm}^{2}$. Figure 1 shows the SEM images of craters produced at $N=1,5$ and 25 with increasing fluence levels ranging from $F_{0}=0.98 \mathrm{~J} / \mathrm{cm}^{2}$ to $40.8 \mathrm{~J} / \mathrm{cm}^{2}$ with 6.7 ps laser source. In general, the diameter of a crater increases with increasing fluence indicating that the tail of the Gaussian pulse becomes intense enough to cause material modification. This effect is intensified if the number of pulses at the same location increases. As shown schematically on the topright image of Fig. 1, each subsequent pulse arrives after about $3.9 \mathrm{~ms}$. Therefore, the laser irradiated area is resolidified before the next pulse arrives, because the typical lifetime of melt from ultrashort pulse irradiation is in $\mu$ s time period [10]. The absorption of consecutive laser pulses by the sample depends on the resolidified surface structures after previous pulses. As a result, similar type of surface morphology dominates with increasing number of pulses at low fluence values as can be seen from Fig. 1 for $F_{0}=0.98 \mathrm{~J} / \mathrm{cm}^{2}$ and $3.6 \mathrm{~J} / \mathrm{cm}^{2}$. At high fluence values $\left(F_{0}>10 \mathrm{~J} / \mathrm{cm}^{2}\right)$, crater morphology is determined mainly by the hydrodynamic motion of the melt. Craters produced at high fluence levels appears more melty in form of frozen splashes as shown in Fig. 1 for $F_{0}=40 \mathrm{~J} / \mathrm{cm}^{2}$.
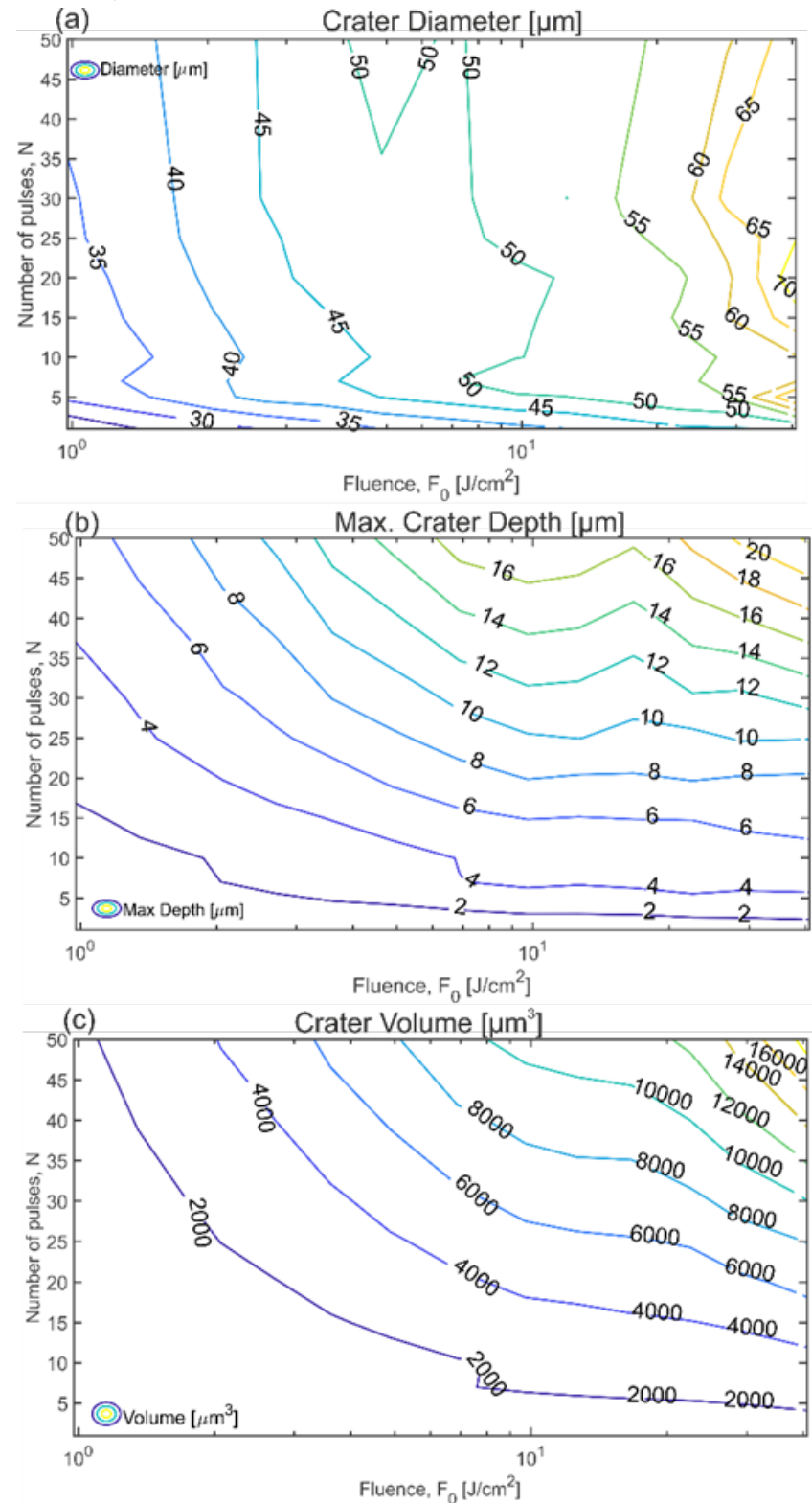

Fig. 2 Contour plots of crater dimension on $\mathrm{Zn}$ at $\tau=7 \mathrm{ps}$ as a function of incident number of laser pulses $N$ and peak fluence $F_{0}$; (a) diameter, (b) maximum depth, (c) volume. 


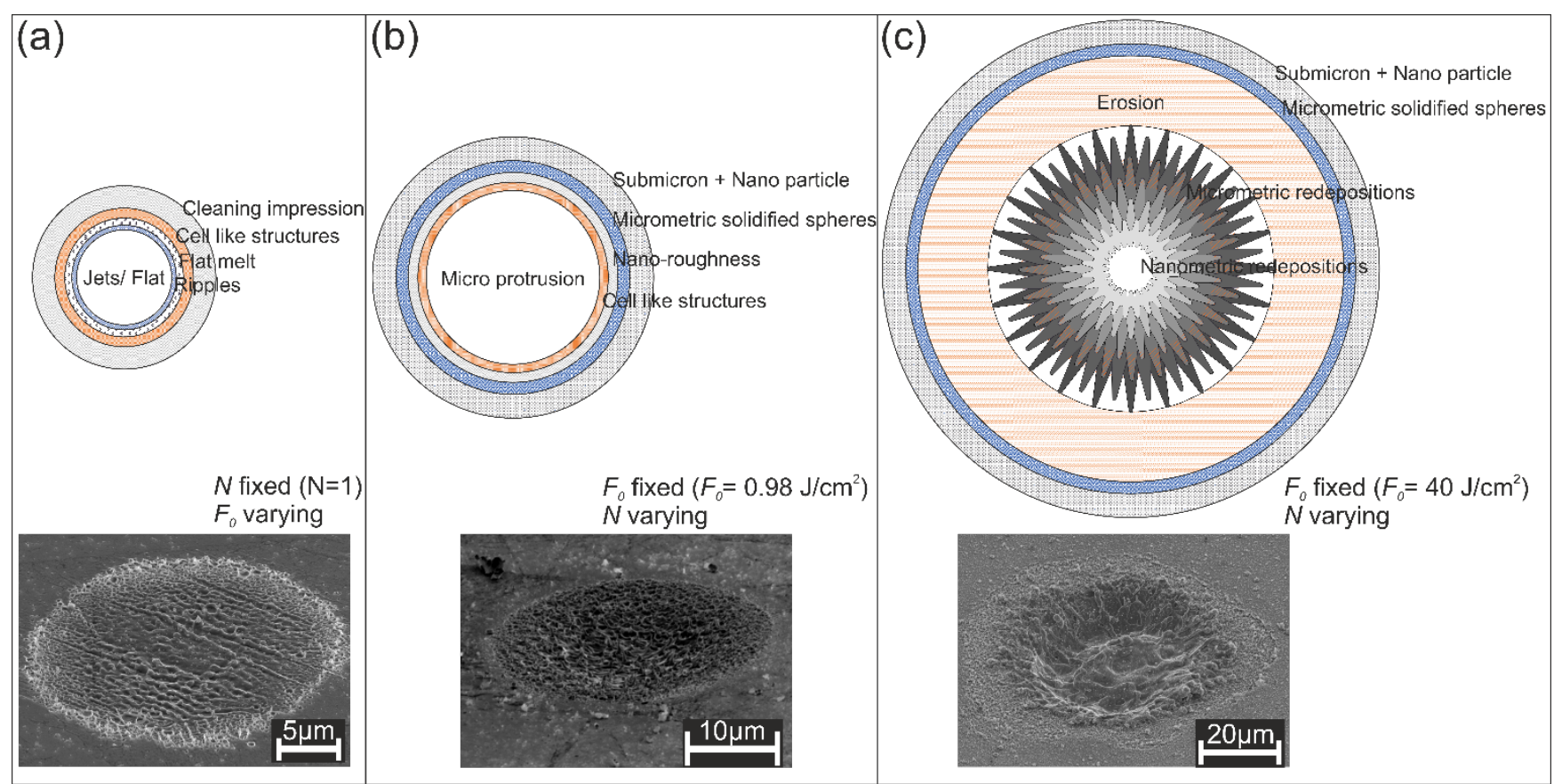

Fig. 3 Schematic representation of morphological features observed on Zn after single and multiple 7 ps laser pulse processing along with the SEM image of the corresponding representative craters at the bottom. (a) $N=1, F_{0}=0.98-40 \mathrm{~J} / \mathrm{cm}^{2}$ with a SEM image (tilted $70^{\circ}$ ) of a representative crater processed at $F_{0}=6.87 \mathrm{~J} / \mathrm{cm}^{2}$, (b) $N=1-50, F_{0}=0.98 \mathrm{~J} / \mathrm{cm}^{2}$ with a SEM image (tilted $70^{\circ}$ ) of a representative crater processed at $N=25$ and (c) $N=1-50, F_{0}=40 \mathrm{~J} / \mathrm{cm}^{2}$ with a SEM image (tilted $50^{\circ}$ ) of a representative crater processed at $N=20$. All schematic diagrams are in same scale.

Although we do not observe the well-known saturation limit of depth and diameter of the ablated craters [11] within our experimental conditions, we see that the dimension of craters depends both on the laser fluence and the applied number of pulses and increases with increasing $F_{0}$ and $N$. Fig. 2 shows the contour plots of the diameter, maximum depth and volume of the ablated craters as a function of $F_{0}$ and $N$. As can be concluded from this figure, the rate of increase in depth and volume is rapid up to $F_{0}=10 \mathrm{~J} / \mathrm{cm}^{2}$ and slows down afterwards with increasing $F_{0}$ or $N$. On the other hand, the diameter is affected more by the change in $F_{0}$ than the change in $N$. However, the aim of the paper is about investigating resolving crater morphology, not about crater dimensions. Therefore, only changes observed in the morphology with $F_{0}$ and $N$ is described in the remaining of the paper.

Fig. 3(a) shows the evolution of different surface structures at different laser fluence levels for $N=1$. At the lowest fluence level and single pulse processing, evident signs of melting and phase explosion are visible in terms of exploding bubbles and jets with spherical endings (see also Fig. 11(a)). Outside the boundary of the actively modified area (i.e. outside the "cell like structures" in Fig. 3(a)), some form of "cleaning" is observed within a radius of $4 \mu \mathrm{m}$. With increasing fluence, a flat modified area bounded by "ripples" increases at the center of the crater and "cell like structures" agglomerate at the edge, see Fig. 3(a). These ripples develop crests towards the edge of the crater with increasing fluence indicating melt expulsion by recoil pressure exerted by the ablation plume, see Fig. 1 bottom-left image and Fig. 10(a). For single pulse processing, depth of the ablated crater lies within $250 \mathrm{~nm}$ to $650 \mathrm{~nm}$ for a forty fold increase in laser fluence, stemming mainly from the melt expulsion rather than the ablation itself. On the other hand, the diameter of the modified area increases from about $20 \mu \mathrm{m}$ to about 47 $\mu \mathrm{m}$ with increasing laser fluence. Presence of liquid droplets and redeposited metal particles around the crater is not common for $N=1$ and submicron droplets, if any, lie within 4 $\mu \mathrm{m}$ and $7 \mu \mathrm{m}$ around the crater edge for lowest and highest fluence respectively. The complete picture of above mentioned feature evolution is shown schematically in Fig. 3(a).

At the lowest fluence with increasing number of pulses, we see both the evolution of depth and diameter of the craters as shown in Fig. 3(b). It is observed that the mean size of the microprotrusions gets larger and becomes more sparsely distributed in the spot center with increased pulse numbers. Within the crater, nanometric particle redeposition is higher for lower number of pulses than for higher number of pulses. On the other hand, redistribution of submicron and microparticles around the laser spot becomes more prominent with increasing pulse number and redeposits as far as $\approx$ $17 \mu \mathrm{m}$ from the edge of the crater for $N=50$, see e.g. SEM image in Fig. 3(b) for $N=25$. This suggests that the plasma

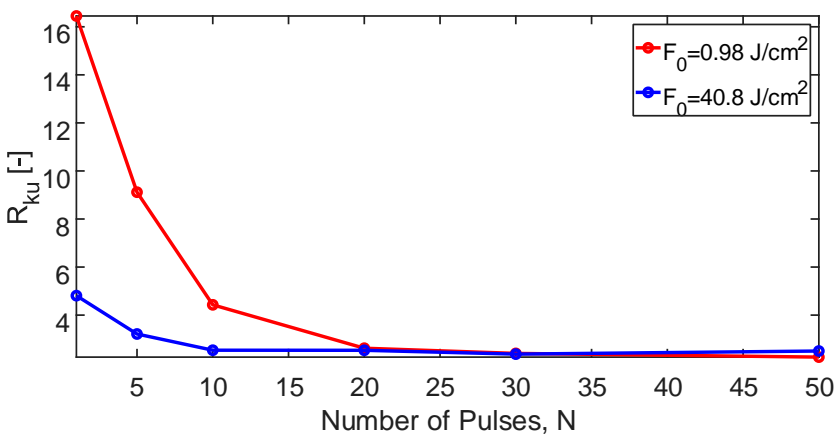

Fig. 4 Evolution of kurtosis roughness $R_{k u}$ in the crater as a function of number of pulses $N$ on $\mathrm{Zn}$ for $\tau=7$ ps. 
72

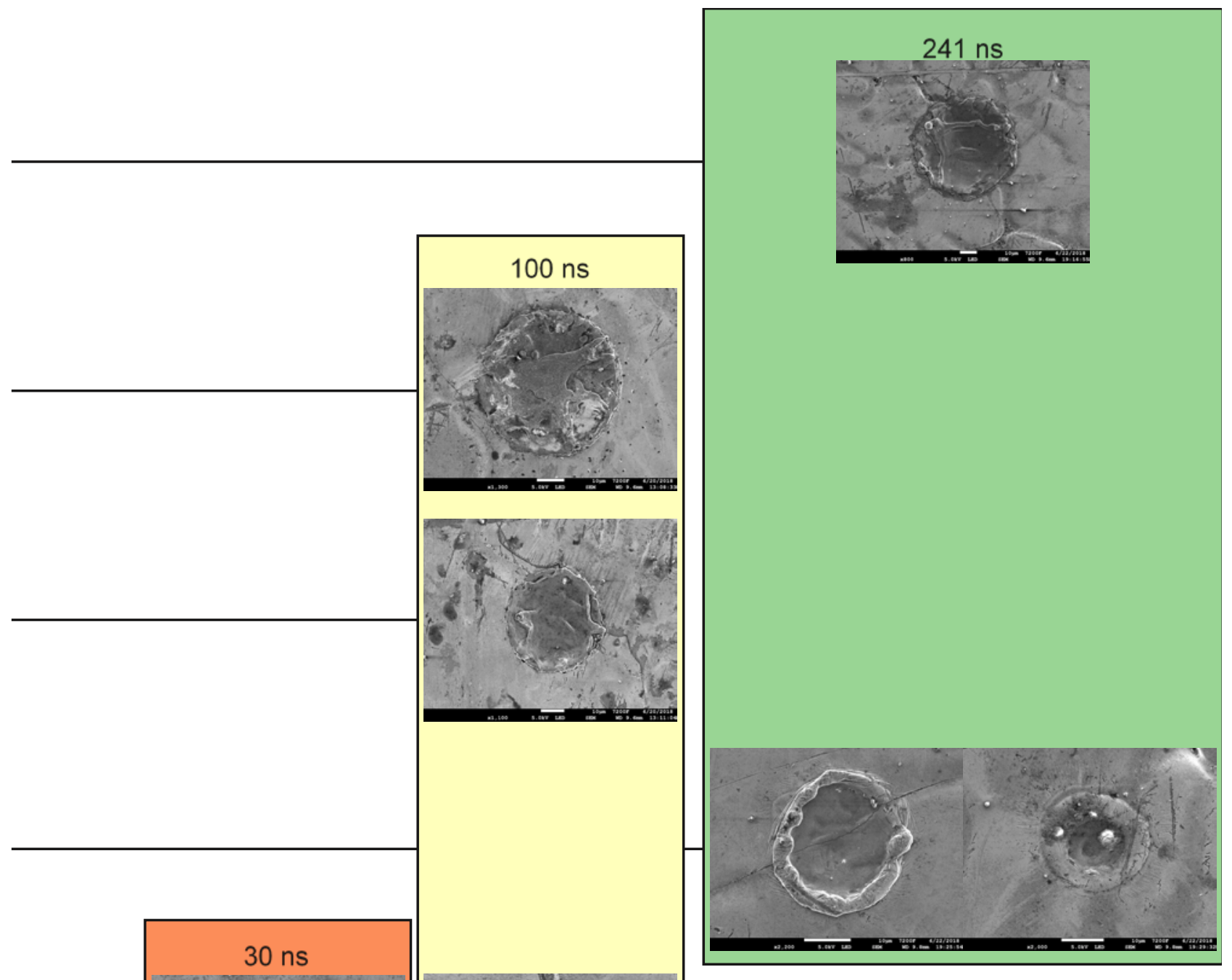

19

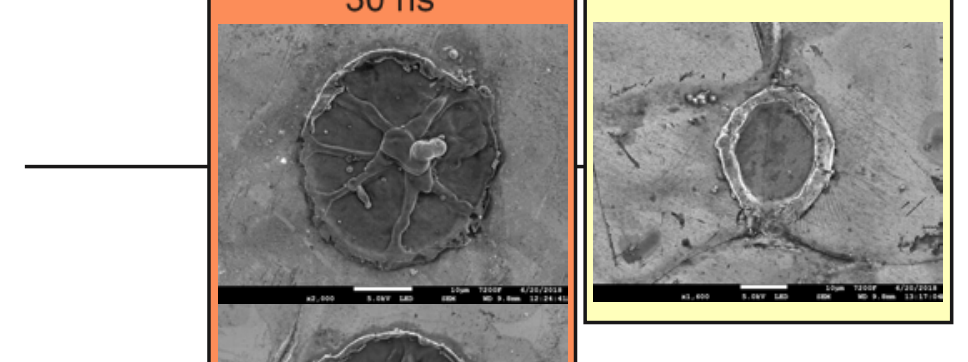

17

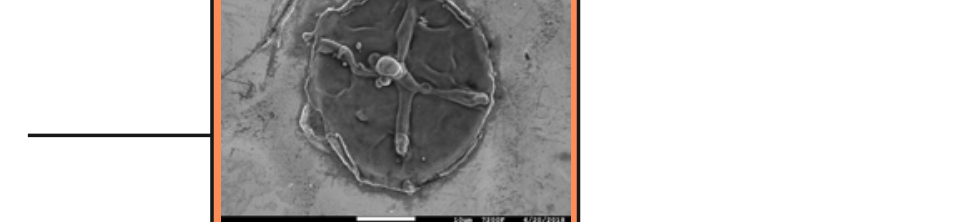

12

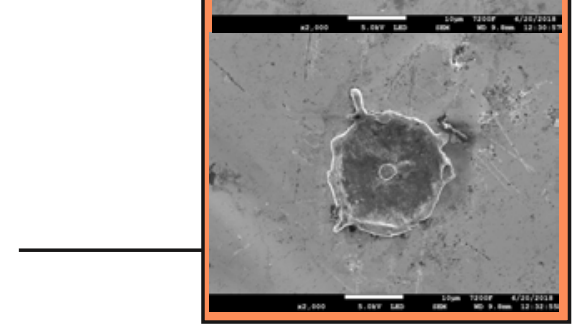

Fig. 5 SEM images (top view) of zinc coated surface irradiated at different laser peak fluence $F_{0}$ levels at $N=1$ for 30,100 and 241 ns laser pulses. White bar in the SEM images denotes $10 \mu \mathrm{m}$.

plume expands in greater speed for higher number of pulses than in low number of pulses. This results in less nanoparticle redeposition inside the crater. The microrims also gets thicker and shows evident expulsion and redistribution of melt towards the edge. At $N=5$, peripheral nano-roughness starts to appear in the form of stretched marks and extends up to $6 \mu \mathrm{m}$ from the crater boundary as shown by the "nanoroughness” annular ring in Fig. 3(b). High velocity particles 
from the ablation process impact onto this area resulting in features similar to bubble bursts within the crater. In Fig. $3(\mathrm{c})$, the surface feature evolution is shown for highest fluence value used in this work, as the number of pulses in the same spot increases. Similar to lower fluences, particle redeposition occurs outside the crater boundary. The bigger sized particles fall closer to the crater periphery and the smaller sized particles redeposit further as shown in Fig. 3(c). Also the shapes of the craters become asymmetric as they tend to deviate from the Gaussian shape. Features like erosion, hump, waves and splashes, as seen in the SEM image of Fig. 3(c) around and in the crater respectively, are discussed in details in Section 3.1, 3.2 and 3.3.

In general, the roughness at the bottom of the crater decreases as the number of pulses increases for a given laser fluence. The roughness, at the bottom of the crater, can be measured from CLSM data and is shown in Fig. 4. Since the crater depth increases with increasing number of pulses, we use kurtosis roughness according to JIS B 0601: 2001 (ISO 4287: 1997) standard, which is a dimensionless quantity. It is calculated by dividing the average of the fourth power of the height of each point by the root mean square height within the region of interest (ROI). In this figure, each data point is an average of 10 craters. The limit for decreasing the roughness would be - when a uniform melt has been pushed away from the center of the crater to the edge by the ablation plume, creating a flat crater bottom.

For ns pulse processing, the variations in surface structures, when varying $F_{0}$ and $N$, are less than ps pulse processing as illustrated in Fig. 5. In this case, the surface structures are "only" micrometric jet and a well-defined rim. Although the ablation threshold energy is much higher for ns pulses than for ps pulses, the depths of the craters are higher and the craters are Gaussian shaped with a flat crater bottom irrespective of $F_{0}$ and $N$. For longer pulse durations ( $\tau>1 \mathrm{~ns}$ ), the target material heats up to melting point and to vaporization point following classical heat transfer equation [7]. Significant evaporation of the target material takes place as soon as the incident laser fluence is larger than the specific heat of evaporation. This results in plasma plume formation. At the same time, energy loss through heat conduction to solid creates a molten region. The plasma pushes the melt away from the center of the crater towards the edge. Details of the features like jets and rim, as seen in the SEM image of Fig. 5, are discussed in Section 3.4 and 3.5.

In the following, we focus on the specific features and structures we observed in the short (ns) and ultrashort (ps) laser irradiation of zinc and zinc coated (galvanized) steel surfaces. For $N=1$, as received galvanized steel sample $\left(R_{a}\right.$ $=0.3 \mu \mathrm{m}$ ) is comparable to the pure zinc block. This is because, optical heating is a surface phenomenon and the effect of the substrate can be neglected for a coating thickness of about $8 \mu \mathrm{m}$ or larger. However, the substrate effect becomes prominent for multiple pulses. Therefore, only results of galvanized steel for $N=1$ is discussed in the following subsections.

\subsection{Erosion}

It has been discussed in the previous section that surface modification in form of "cleaning" is observed outside the boundary of active modified area at $N=1$, see Fig. 3(a). Also,

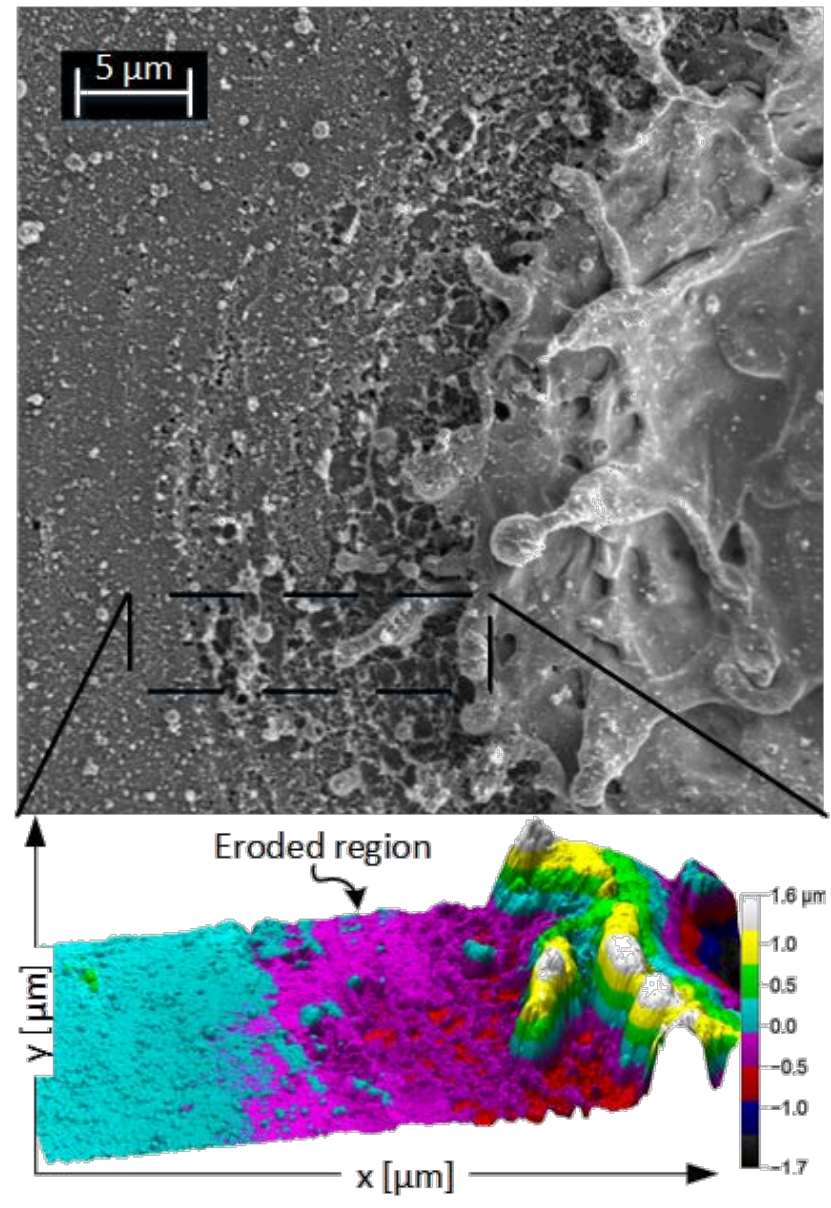

Fig. 6 Erosion around the crater at $N=20, F_{0}=40.8 \mathrm{~J} / \mathrm{cm}^{2}$ on $\mathrm{Zn}$ for $\tau=7 \mathrm{ps}$. Lower image corresponds to AFM height image of the area marked with black rectangle in the upper SEM image.

at low fluence values but $N \geq 5$, nano-roughness starts to appear outside the boundary of the actively modified area as an evident sign of stress from rapid solidification, see Fig. 3(b). Figure 6 shows the sample surface outside the crater for $N=20$ at the highest fluence level used in this work. It appears as if melting and its subsequent liquid has taken place beneath a hard top surface layer, which leaves this hard top surface stretched and shattered in places. This is analogous to riverbank erosion where softer rock underneath is eroded ahead of the harder rock on top. AFM measurements show that the this possible "eroded" area indeed has a depth difference compared to the reference surface and increases towards the crater, being as deep as $100 \mathrm{~nm}$. Figure 7 shows laser beam cross-section at representative fluence levels along with the theoretical melting and evaporation threshold calculated as [12]

$$
F_{t h}^{e}=\frac{\delta \cdot \rho \cdot\left(\left(T_{m}-T_{0}\right) \cdot C_{p}+H_{m}+H_{v}\right)}{A}
$$

where, $\rho, H_{m}, H_{v}, C_{p}, T_{m}, T_{0}, \delta$ and $A$ represent the density of the solid (7140 kg/m $\mathrm{m}^{3}$ [13]), the enthalpy of fusion $\left(111 \times 10^{3} \mathrm{~J} / \mathrm{kg}[14]\right)$, the enthalpy of evaporation $\left(1748 \times 10^{3}\right.$ $\mathrm{J} / \mathrm{kg}$ [14]), specific heat $\left(0.382 \times 10^{3} \mathrm{~J} /(\mathrm{kg} \cdot \mathrm{K})\right.$ [13]), melting point (692.68 K [13]), room temperature (300 K), the energy penetration depth and absorption coefficient, respectively. The total penetration depth, including optical and ballistic electron penetration depth, of about $50 \mathrm{~nm}$ is taken as the energy penetration depth in Eq. (1). Left inset of Fig. 7 


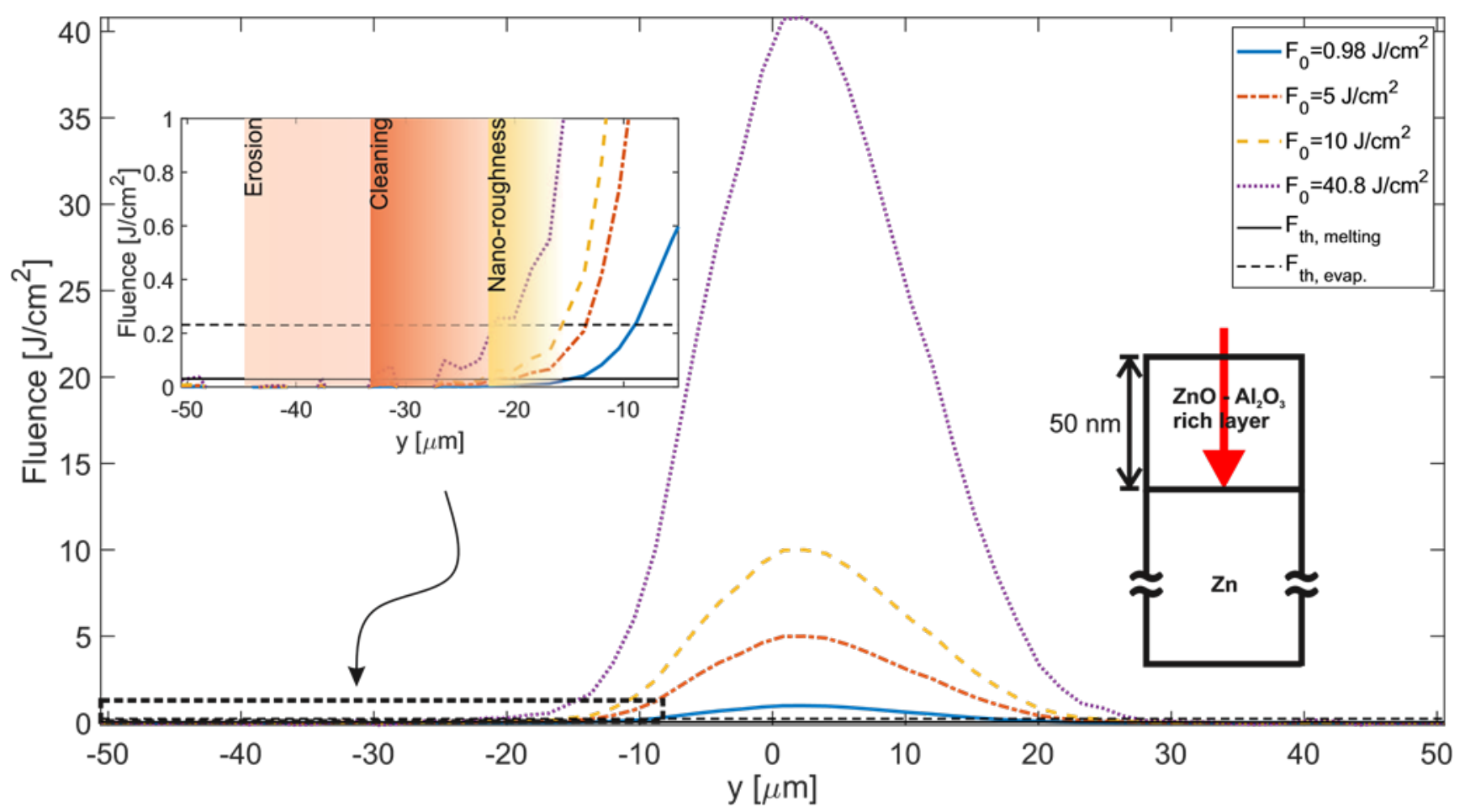

Fig. 7 Laser beam cross-section for different laser fluence levels along with theoretical melting and evaporation threshold according to Eq. (1). Left inset shows the zoomed image of the tail of the Gaussian profile. Right inset is a schematic representation of native oxide layers on bulk zinc, where red line indicates laser beam at $1030 \mathrm{~nm}$.

shows the zoomed image of the tail of the Gaussian profile indicating the extent of the surface features like cleaning, nano-roughness and erosion. These features appear when the laser fluence crosses the melting threshold. Right inset in Fig. 7 is a schematic representation of native oxide layers. X-ray photoelectron spectroscopy (XPS) analysis on the virgin sample revealed the presence of native oxide layers as thick as $50 \mathrm{~nm}$. Both $\mathrm{ZnO}$ and $\mathrm{Al}_{2} \mathrm{O}_{3}$ are transparent to infra-red wavelength. Thus, the laser beam penetrates through the oxide layer without significant absorption in the oxides. Moreover, both these oxide layers are considerably harder and possess higher melting point than $\mathrm{Zn}$. Therefore, the tails of Gaussian beam at high fluence level becomes intense enough to melt $\mathrm{Zn}$ but not the corresponding oxides. This results in surface erosion as depicted in Fig. 6.

\subsection{Hump}

It has been observed from the cross-sections of ablated craters, obtained from CLSM measurement (see Fig. 1 bottom-right image and Fig. 3(c)), that the craters are Gaussianshaped for all $N$ for fluence level up to $F_{0} \leq 10 \mathrm{~J} / \mathrm{cm}^{2}$, but start to deviate from this shape at higher fluence levels. At

Table 2 Thermo-physical properties of Zinc [13] and Aluminum [18].

\begin{tabular}{lll}
\hline Parameter & Zn & $\mathrm{Al}$ \\
\hline Latent Heat of Melting [J/g] & 100.9 & 388 \\
Latent Heat of Vaporization [J/g] & 1782 & 10800 \\
Absorptivity @ 1030 nm [\%] & 42.11 & 4.03 \\
Optical Penetration depth @ 1030 nm [nm] & 25.4 & 8.45 \\
Theoretical evaporation threshold (Eq.(1)) & 0.09 & 0.67 \\
[J/cm²] & &
\end{tabular}

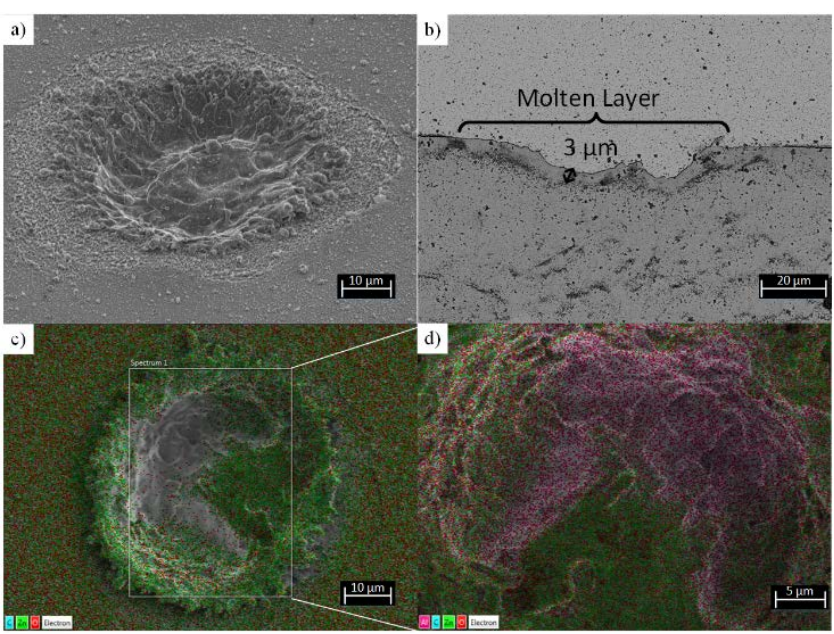

Fig. 8 (a) Ablated crater at $N=20, F_{0}=40.8 \mathrm{~J} / \mathrm{cm}^{2}$ with 'hump' in the center and surface modification (erosion) around the crater (tilted $60^{\circ}$ ) on $\mathrm{Zn}$ for $\tau=7$ ps. (b) Backscattered electron (BSE) image of cross-section of a similar crater ablated at $N=50, F_{0}=$ $40.8 \mathrm{~J} / \mathrm{cm}^{2}$. (c) EDS layered image of a crater ablated at $N=50$, $F_{0}=40.8 \mathrm{~J} / \mathrm{cm}^{2}$, and (d) EDS layered image of the area marked with white rectangle in (c).

the maximum fluence value used in this work, the crater shape differs significantly from a Gaussian profile, showing a "hump" in the center surrounded by a groove (see Fig. 8(a)). A similar bulging shape was also observed for Si [15]. At $N=50$, for example, the hump extends over $1 / \mathrm{e}^{2}$ beam diameter from the center of the crater. The height difference is $\sim 10 \mu \mathrm{m}$ from the deepest point.

The hump formation may be attributed to the segregation of aluminum from the solid solution ( $\mathrm{Zn}-\mathrm{Al}$ ) during ablation. There is no intermetallic formation in the $\mathrm{Zn}-\mathrm{Al}$ solid solution, which means the interaction between $\mathrm{Al}$ and $\mathrm{Zn}$ atoms 
is weak [16]. Comparing the thermal and physical properties of $\mathrm{Zn}$ and $\mathrm{Al}$ in Table 2, $\mathrm{Zn}$ ablation rate is expected higher than Al. Melting of the zinc is expected with the fluence levels applied in this study. As the zinc is ablated, the aluminum tends to be clustered and supersaturated in the remaining molten $\mathrm{Zn}$ resulting in a new thin $\mathrm{Zn}-\mathrm{Al}$ solid solution layer. This process is repeated as the number of the laser pulse increased since $\mathrm{Al}$ is highly reflective at $1030 \mathrm{~nm}$ wavelength. As a result, the accumulation of new $\mathrm{Zn}-\mathrm{Al}$ solid solution may become a layer as shown in Fig 8(a) and (b). This also leads to lower ablation rate than the low fluence regime $\left(F_{0}\right.$ $<10 \mathrm{~J} / \mathrm{cm}^{2}$ ) [17]. Figure 8(b) shows a cross-section of the crater processed with 50 laser pulses with $F_{0}=40.8 \mathrm{~J} / \mathrm{cm}^{2}$. From this figure, the melt layer thickness is estimated to be around $3 \mu \mathrm{m}$ with higher aluminum content.
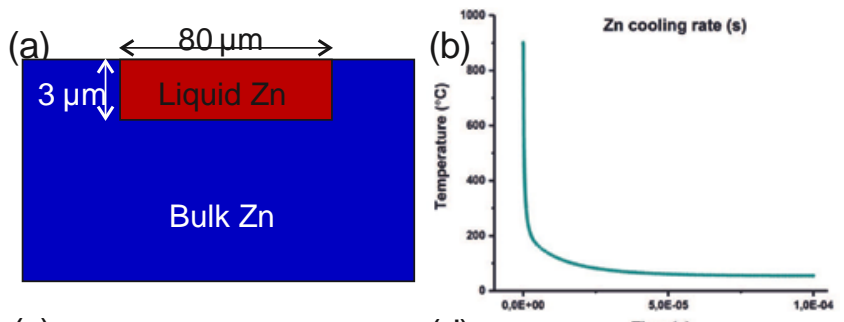

(c)

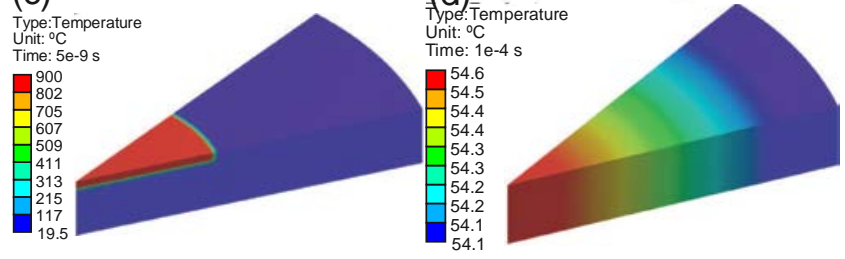

Fig. 9 (a) Schematic diagram of the ANSYS model representing a liquid melt within bulk Zn. (b) Cooling rate of $\mathrm{Zn}$ obtained from ANSYS model where (c) initial boundary condition at $5 \mathrm{~ns}$ and (d) temperature at $100 \mu \mathrm{s}$.

A 3D transient thermal FEM analysis was performed in ANSYS 19.0 as shown in Fig. 9. For analysis, a 30 symmetric model was meshed with solid187 element type. An initial temperature boundary condition of $900^{\circ} \mathrm{C}\left(T_{\text {evap }}\right)$ was applied to the $\mathrm{Zn}$ melt of a thickness of $3 \mu \mathrm{m}$, see Fig. 9(c). The cooling rate was calculated by taking into account the heat conduction to the surrounding material, and combined heat loss due to convection and radiation. It was found that $\mathrm{Zn}$ melt cooled down to $54^{\circ} \mathrm{C}$ in $100 \mu$ s as shown in Fig. 9(b) and (d). Since zinc has a positive temperature coefficient of surface tension [15], this hump could result from the thermocapillary flow of the superheated molten $\mathrm{Zn}$.

Subsequent EDS analyses were performed, and the results are shown in Fig. 8(c), in which the green denotes the presence of $\mathrm{Zn}$ and pink denotes the presence of Al. The groove around the hump is too deep for the EDS detector to detect any elemental composition. Therefore, area marked in white rectangle is analyzed for retrieving a better compositional map as shown in Fig. 8(d). From these two images, it can be concluded that, $\mathrm{Zn}$ is present in the hump whereas $\mathrm{Al}$ is segregated from the melt and deposited in the groove around the hump.

\subsection{Waves and Instabilities}

Although the craters are disc-shaped at low fluence, they become more splash-dominated with increasing $F_{0}$ and $N$, see Fig. 1. Ripples with crests for $N=1$ (Fig. 10 (a)) turns into splats with fingers (Fig. 10 (b)) for higher number of pulses at the center of the crater. At $F_{0}<10 \mathrm{~J} / \mathrm{cm}^{2}$ and $N \geq 1$, splash fingers do not develop completely. However, for $F_{0} \geq$ $10 \mathrm{~J} / \mathrm{cm}^{2}$, these fingers "stack" over each other in an alternating manner as shown in Fig. 3(b). Also, these melt splash fingers do not develop radially, but rather randomly, around the crater center. The spherical fingertip detaches from the

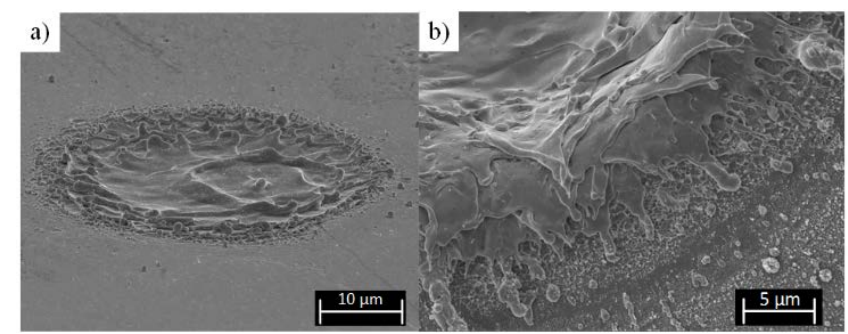

Fig. 10 (a) Ablated crater at $N=1, F_{0}=40.8 \mathrm{~J} / \mathrm{cm}^{2}$ (tilted 70 $)$ on Zn for $\tau=7$ ps. (b) Splash fingers over the crater rim at $N=25$; $F_{0}=40.8 \mathrm{~J} / \mathrm{cm}^{2}$ (top view).

finger and redeposits as micrometric spheres around the crater. Eventually by melt expulsion, stacked splash fingers form a rim around the crater at high fluence levels and/or number of laser pulses.

Increasing the laser fluence results in a transition to a molten region with waves and fingers (see e.g. the single pulse results in Fig. 1). The final morphology features solidified layers of the ablated materials with clear azimuthal instabilities. In such a regime, a part of the laser energy absorbed by the material leads to a solid-liquid phase change. Meanwhile, the plasma formation generates a pressure gradient on the surface of the thin molten film. This results in a flow towards the edge of the crater. We hypothesize the "layered” morphology is a consequence of Kelvin-Helmholtz instability [19], due to the shear at the interface of the molten material and the plasma/vapor. The Kelvin-Helmholtz waves grow in the radial direction, which leads to azimuthal transverse modulations due to the Rayleigh-Taylor instabilities [19], i.e. the edge of the accelerating waves become unstable and forms finger-like jets. These finger-like jets further stretch and in some cases break up because of Rayleighplateau instability [20], redepositing micrometric spheres around the crater. A similar phenomenology has previously been observed in spray formation [21] and deformation of a droplet in a gas stream [22]. Nevertheless, a direct experimental observation is yet to be provided in the future studies, using ultra-high speed imaging.

The hydrodynamical phenomena explained above are damped by surface tension and the viscosity of the melt and are eventually ceased due to re-solidification. A simple scaling reveals that the heat conduction time scale $\left(L^{2} \rho C_{p} / k\right.$, where $L, \rho, C_{p}$ and $k$ are the characteristic length scale, density, heat capacity, and heat conductivity, respectively) is smaller or comparable to the visco-capillary time scale $(L \mu / \sigma$, where $L, \mu$, and $\sigma$ are a characteristic length scale, viscosity, and surface tension, respectively) as well as the flow time scale ( $\mu L^{2} / P_{a v g} h_{m e l t}$, where $P_{a v g}$ and $h_{m e l t}$ are the plasma pressure and the melt thickness, respectively). Therefore, the melt instabilities are retarded while developing due to the solidification, as is clear in the SEM pictures of the final morphologies (Fig. 10). 
Increasing the pulse number results in a more complicated final morphology. On the one hand, the crater depth grows and on the other hand, a larger number of fine spherical residuals (solidified droplets) are created around the crater. The latter might be due to the complex initial shape of the target for the subsequent pulses.

\subsection{Jets}

Two types of jets can be observed for picosecond and nanosecond pulsed processing, which only occurs for $N=1$ and was not observed for higher number of pulses, see Fig. 11. In ps laser irradiation, jets with spherical endings cover the laser irradiated zone at low fluence values as shown in Fig. 11(a). Similar features were observed by Oboňa et al. for $\mathrm{Cu}$ surfaces processed with the same ps-pulsed laser source $[23,24]$. These jets protrude from the reference surface $780 \mathrm{~nm}$ on average. This results in a subsequent minima or valley as low as $480 \mathrm{~nm}$ from the reference surface. Of course, this is neither ablated nor removed material. Rather these maxima and minima are a consequence of the rapid solidification of the displaced melt due to inertia, viscous and capillary forces acting during the formation of the bubble in the propagating thin liquid layer [23]. The size of the spherical tip is bigger in the center of the crater than on the edges.
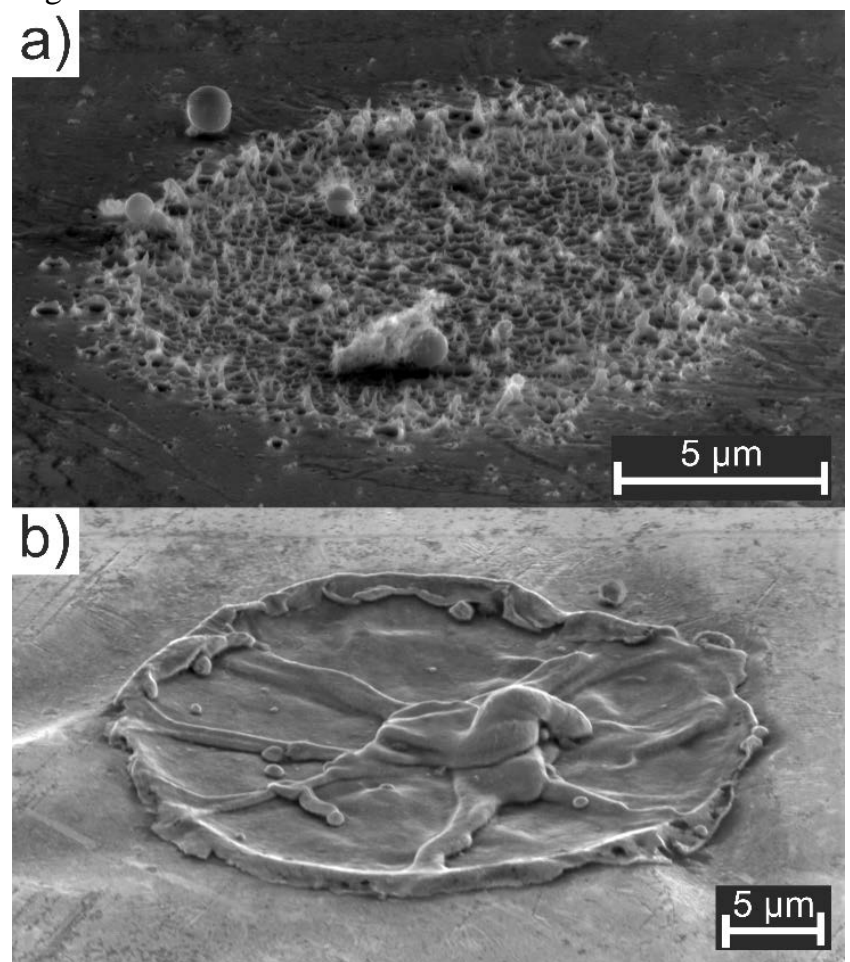

Fig. 11 SEM images of (a) crater processed at $N=1 ; F_{0}=0.99$ $\mathrm{J} / \mathrm{cm}^{2}$ with $7 \mathrm{ps}$ laser pulse featuring jets with spherical endings in the center of the crater and micrometric redeposition (tilted $60^{\circ}$ ) on $\mathrm{Zn}$, and (b) crater processed at $N=1 ; F_{0}=19.7 \mathrm{~J} / \mathrm{cm}^{2}$ with 30 ns laser pulse showing jet with micrometric spherical ending (tilted $65^{\circ}$ ) on $\mathrm{Zn}$ coated sample.

The second type of jet is observed in ns laser processing for relatively short pulse duration ( $<50 \mathrm{~ns}$ ). Longer pulse duration in ns regime results in a complete melt redistribution towards the edge of the crater creating rims as high as 1 $\mu \mathrm{m}$. In Fig. 11(b), such a jet is shown on galvanized steel sample, which resembles a wheel with spokes. The jet rises more than $2 \mu$ m over of the reference surface. The ellipsoidal tip is extended over $5 \mu \mathrm{m}$ along the major axis and $2.5 \mu \mathrm{m}$ along the minor axis. The total number of spokes is seven and the height of the spokes from the crater bottom is more than $0.5 \mu \mathrm{m}$. This may result from the increasing surface tension of $\mathrm{Zn}$ with temperature as mentioned earlier. Similar kind of micrometric jet was also observed for gold and was attributed to the liquid material being "sucked up" into the crater center due to the drop of ablation pressure behind the ablation plume [25].

\subsection{Rim}

Ablation of the material results in an ablation plume with a melt layer as a result of the residual heat energy within the target during and/or after the pulse but prior to resolidification. This plume is associated with internal and external shock waves [26]. These shock waves, i.e. plasma pressure pushes the melt outwards as the pressure gradient is extremely high at the plasma-air interface. If the lifetime of the melt layer is longer than the plasma pressure generation time, the melt is pushed outwards more strongly [27]. Figure 12(a) shows a crater processed with 50 ps laser pulses. It can be seen that the rim formed around the crater has evident signs of melt expulsion with each new pulse arriving on the same location. On the other hand, Fig. 12(b) shows a typical rim around a ns pulsed laser processed crater analogous to a Napoli pizza. The maximum depth of this crater is about 2.3 $\mu \mathrm{m}$, whereas the rim height is about $1.6 \mu \mathrm{m}$. Within the
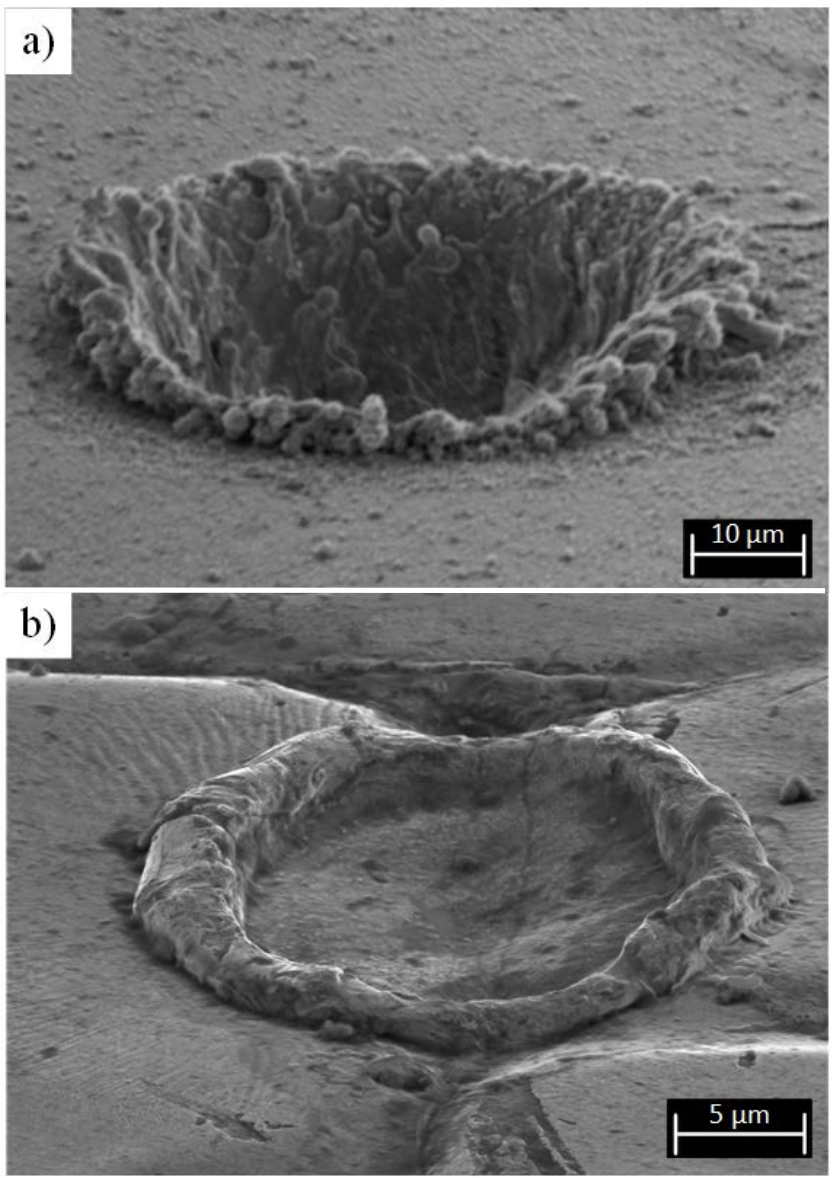

Fig. 12 SEM image of (a) crater processed at $N=50, F_{0}=40.8$ $\mathrm{J} / \mathrm{cm}^{2}$ (tilted $70^{\circ}$ ) on Zn for $\tau=7 \mathrm{ps}$, (b) crater (tilted $65^{\circ}$ ) processed on a $\mathrm{Zn}$ coated sample by a $100 \mathrm{~ns}$ laser pulse at $N=1$; $F_{0}=19.2 \mathrm{~J} / \mathrm{cm}^{2}$. 
boundary of our experimental conditions, ns laser pulses always results in a crater with a rim. At low fluence levels, there is either transient surface melting and resolidification or formation of a crater with a rim. As the fluence and/or number of pulses is increased, the rim height increases.

The height of the rim $h_{\text {rim }}$ can be estimated as [28]

$$
h_{\text {rim }} \approx \frac{\rho_{\text {avg }} \cdot h_{\text {melt }}}{\mu \cdot D^{2}}
$$

where $\rho_{\text {avg }}$ is the average plasma pressure, $h_{\text {melt }}$ is the melt layer thickness, $D$ is the ablated crater diameter and $\mu$ is the viscosity As the laser fluence is increased, the plasma pressure and temperature also increases with the melt temperature. Therefore, an increase in the rim height is observed with increasing fluence both in ps and ns pulse durations. As can be observed in Fig. 2, the depth of the crater also increases with fluence. This implies that the rim height increases with increasing crater depth. This is found to be true for ns laser processing of Zn. Figure 13 shows the height image of ablated craters on galvanized steel at similar processing conditions, but at different laser pulse durations. The rim around the crater is more pronounced in longer pulse durations than shorter ones because of the slower decrease of temperature, as well as higher initial velocity of the ns plume than ultrashort pulse plumes [26]. Thus, the rim around the crater gets higher as the laser material interaction time increases.

\section{Conclusion}

Within the boundaries of our experimental conditions, laser ablation of $\mathrm{Zn}$ is a melt dominated process. In spite of that, the crater morphology of ps- and ns-ablated craters differ substantially. The shorter lifetime of the plasma plume as well as liquid melt makes the difference. Three different microscopy techniques were utilized to investigate the resulting surface structures.

- At the lowest fluence in the ps regime, the laser irradiated zone is covered with jets with spherical endings. In contrast, ns laser pulses modify the surface either through transient melting and resolidification or by creating a crater with a well-defined rim.

- There is a limit of laser peak intensity, below which the shape of the crater follows the Gaussian shape of the incoming beam intensity profile. If the intensity is high enough, the composition of the $\mathrm{Zn}$ alloy changes locally due to supersaturation of $\mathrm{Al}$ at the surface.

- As the number of pulses increases, the previously formed microstructures affect the later formed surface structures in an accumulative manner.

Therefore, depending on the requirements of the surface texture for a given application, either ps- and/or ns-pulsed laser sources can generate different kinds of textures resulting from resolidification.

\section{Acknowledgments}

The authors would like to acknowledge the financial support of Tata Steel Nederland Technology BV. We would also like to thank ing. E.G. de Vries for AFM measurements, L. Capuano for the help with SEM and EDS measurements, and Dr. R. Pohl, Dr. B. Pathiraj, Dr. ir. T.C. Bor of the University of Twente for their fruitful discussions on this topic.

\section{References}

[1] F. C. Porter: "Corrosion resistance of zinc and zinc alloys”, (Publisher, CRC Press, 1994).

[2] T. H. Muster, W. D. Ganther and I. S. Cole: Corros. Sci., 49, (2007), 2037.

[3] B. Qian and Z. Shen: Langmuir, 21, (2005), 9007.

[4] H. Zeng, W. Cai, Y. Li, J. Hu and P. Liu: J. Phys. Chem. B, 109, (2005), 18260.

[5] V. Svetlichnyi and I. Lapin: Russ. Phys. J+, 56, (2013), 581.

[6] S.-S. Wellershoff, J. Hohlfeld, J. Guddë and E. Matthias: Appl. Phys. A: Mater. Sci. Process., 69, (1999), S99.

[7] B. N. Chichkov, C. Momma, S. Nolte, F. Von Alvensleben and A. Tünnermann: Appl. Phys. A: Mater. Sci. Process., 63, (1996), 109.

[8] A. Semerok, C. Chaleard, V. Detalle, J.-L. Lacour, P. Mauchien, P. Meynadier, C. Nouvellon, B. Salle, P. Palianov, M. Perdrix and G. Petite: Appl. Surf. Sci., 138, (1999), 311.

[9] L. Ang, Y. Lau, R. Gilgenbach, H. Spindler, J. Lash and S. Kovaleski: J. Appl. Phys., 83, (1998), 4466.

[10] M. Garcia-Lechuga, J. Solis and J. Siegel: Appl. Phys. Lett., 108, (2016), 171901.

[11] T. Delgado, D. Nieto and M. T. Flores-Arias: Opt. Laser Eng., 86, (2016), 29.

[12] R. Pohl, C. Visser, G. R. B. E. Römer, C. Sun and D. Lohse: J. Laser Micro/Nanoengin., 10, (2015), 154.

[13]F. C. Porter: "Zinc handbook: Properties, Processing, and Use in design”, (Publisher, CRC Press, 1991).

[14]L. Cabalin and J. Laserna: Spectrochim. Acta, Part B, 53, (1998), 723.

[15] J. Bonse, K.-W. Brzezinka and A. Meixner: Appl. Surf. Sci., 221, (2004), 215.

[16]Ž. Skoko, S. Popović and G. Štefanić: Croat. Chem. Acta, 82, (2009), 405.

[17]S. Ruotsalainen, P. Laakso, R. Penttilä and H. Pantsar: Proc. 11th NOLAMP Conf. in Laser Processing of Materials, Lappeenranta, (2007).

[18] R. Le Harzic, D. Breitling, M. Weikert, S. Sommer, C. Föhl, S. Valette, C. Donnet, E. Audouard and F. Dausinger: Appl. Surf. Sci., 249, (2005), 322.

[19] S. Chandrasekhar: "Hydrodynamic and hydromagnetic stability”, (Publisher, Courier Corporation, 2013).

[20]J. Eggers and E. Villermaux: Rep. Prog. Phys., 71, (2008), 036601.

[21]P. Marmottant and E. Villermaux: J. Fluid Mech., 498, (2004), 73.

[22] M. Jalaal and K. Mehravaran: Phys. Fluids, 26, (2014), 012101.

[23]J. V. Oboňa, V. Ocelík, J. Rao, J. Skolski, G.R.B.E. Römer, A. Huisin’t Veld and J. T. M. De Hosson: Appl. Surf. Sci., 303, (2014), 118.

[24] J. V. Oboňa, V. Ocelík, J. Hosson, J. Skolski, V. Mitko and G.R.B.E. Römer: "Surface effects and contact mechanics X : computational methods and experiments”, (Publisher, WIT Press, 2011).

[25]A. Otto, H. Koch, R. G. Vázquez, Z. Lin, and B. Hainsey: Physcs. Proc., 56 , (2014), 1315.

[26] A. Miloshevsky, S. S. Harilal, G. Miloshevsky and A. Hassanein: Phys. Plasmas, 21, (2014), 043111. 
[27]A. Ben-Yakar, A. Harkin, J. Ashmore, R. L. Byer and H. A. Stone: J. Phys. D: Appl. Phys., 40, (2007), 1447.

[28]P. Duggal: “An experimental study of rim formation in single-shot femtosecond laser ablation of borosilicate glass”, (Publisher, UT Austin, 2006). (Master’s thesis)

(Received: June 26, 2018, Accepted: October 7, 2018) 